\title{
1 Krankenkassen im Sog der Digitalisierung
}

\author{
Helmut Gerhards
}

\section{Digitalisierung im Kontext des Gesundheitswesens}

Die Digitalisierung durchdringt aktuell alle Lebensbereiche unserer Gesellschaft und verändert immer mehr Branchen und Unternehmen. Ähnlich wie in Gesellschaft und Wirtschaft durchlebt das Gesundheitswesen in Deutschland derzeit die Transformation zu digitalen Prozessen. Gewachsene Strukturen werden dadurch verändert und zum Teil neu gestaltet. Aber auch neue Unternehmen, die mit digitalen Geschäftsmodellen groß geworden sind, erweitern ihre Geschäftsfelder und drängen in den Gesundheitsmarkt. Gerade im Bereich der (Gesundheits-)Dienstleistungen haben digitale Technologien in der jüngsten Vergangenheit enorme Produktivitäts-/ und Gewinnsteigerungen möglich gemacht. Der Gesundheitsmarkt bietet ein riesiges Potenzial an weiteren Ideen, digitalen Geschäftsmodellen und Produkten mit ihren Implikationen auf die Leistungsträger (u.a. Kassenlandschaft).

Digitalisierung kann also ein Motor für Wachstum und Wohlstand sein. Die Information - sozusagen der Treibstoff der IT-Transformation-, versetzt das Unternehmen in die Lage - und hier insbesondere das Management-, schneller fundierte Entscheidungen zu treffen. Es gilt also die relevanten Informationen aus der Masse an Big Data zu gewinnen und durch deren Identifikation, vorhandene Potenziale zu heben. Dazu bedarf es jedoch intelligenter Analyseverfahren. Sie bilden einen wichtigen Bestandteil einer erfolgreichen IT-Transformation. Umfangreiche Datenbestände schaffen damit die Grundlage, innovative Produkte und Services für Kunden zu entwickeln. Dies gilt analog für die Beziehungen eines Unternehmens zu seinen Geschäftsund Kooperationspartnern. Die so entstehende stärkere Integration von Kunden und 
Partnern ist ein Differenzierungsmerkmal im Wettbewerb und eine Chance, die eigene Marktposition zu sichern und auszubauen.

Das trifft per se in gleichem Maße auch auf den Krankenversicherungssektor zu. Eine volldigitale Krankenversicherung - nach dem Beispiel von Oscar in den USA - ist auch in Deutschland grundsätzlich vorstellbar. Der Rechts- und Ordnungsrahmen der privaten Krankenversicherung würde dies in bestimmten Grenzen sicher zulassen. Über die Auswirkungen dessen auf den Wettbewerb zwischen privater und gesetzlicher Krankenversicherung lässt sich nur spekulieren. Sicher ist jedoch, dass dies eine große gesellschaftspolitisch zu regelnde Aufgabe darstellt.

\section{Einflussfaktoren und Blickwinkel}

Der Einfluss der Digitalisierung auf die Gesundheitsunternehmen und hier im speziellen auf die gesetzliche Krankenversicherung, kann man aus unterschiedlichen Perspektiven betrachten. Die Kassen müssen diese Suggestionen in Bezug auf das eigene Handeln kennen, erkennen und nicht nur im Rahmen der Rechtsvorgaben, berücksichtigen.

Als gewichtigster regelnder Faktor hat der Gesetzgeber eigene Vorstellungen und Interessen (Vgl. auch Bundesfachausschuss der CDU zur E-Health-Strategie Deutschland 2017). Danach sollen die Chancen der Digitalisierung ergriffen werden, wobei der Mensch im Mittelpunkt steht. Der Ausbau der Digitalisierung im bundesdeutschen Gesundheitswesen soll den innovativen Gesundheitsstandort Deutschlang stärken. Der digitale Wandel soll mithilfe von Hightech-Produkten in der Medizintechnik, innovativen Arzneimitteln, neuen Behandlungsmethoden und Versorgungsformen unter Beachtung des Datenschutzes (aber in Maßen!) vollzogen werden. Weitere moderne Ansätze, wie die effektivere Nutzung von Big Data für die Gesundheitsversorgung und Forschung als auch die vernetzte digitale medizinische Versorgung in der Fläche, zeigen die thematische Auseinandersetzung der involvierten gesundheitspolitischen Akteure sowie die dahinter stehende Intention, das Thema digitalisiertes Gesundheitswesen voranzutreiben.

Ebenso verfolgen die Leistungserbringer und weitere Gesundheitsunternehmen am Markt, ihre eigenen Ziele. Diese Unternehmen verfügen darüber hinaus über einen enormen Zeit(Wissens)-Vorsprung, da sich die Branchen der Handels-, Konsumgüter und Dienstleistungsunternehmen schon viel früher mit den vielfältigen Möglichkeiten der Digitalisierung auseinandergesetzt haben (s. Abb. 1).

Darüber hinaus dürfte diesen Unternehmen schon seit langem bekannt sein, dass bisher profitable Geschäftsmodelle kurzfristig durch digitale Innovation wegbrechen können. Unternehmen wie Uber machen deutlich, wie ein funktionierendes Geschäftsmodell in kürzester Zeit durch disruptive, digitale Innovationen herausgefordert werden kann. Diese Unternehmen agieren also aus unterschiedlichen Motiven heraus und fokussieren ihre Nutzenausrichtung in entsprechende Anwendungen und Services für ihre Kunden. Dass diese Interessen mitunter diametral zu den Interessen der Kassen stehen, versteht sich von selbst.

Nicht zuletzt hat der einzelne Versicherte, determiniert aus seinen Grundbedürfnissen (Gesund zu leben, gesund zu werden, mit der Krankheit zu leben), spezielle Kundenerwartungen und Anliegen, die er schnell und unkompliziert adressieren möchte. 


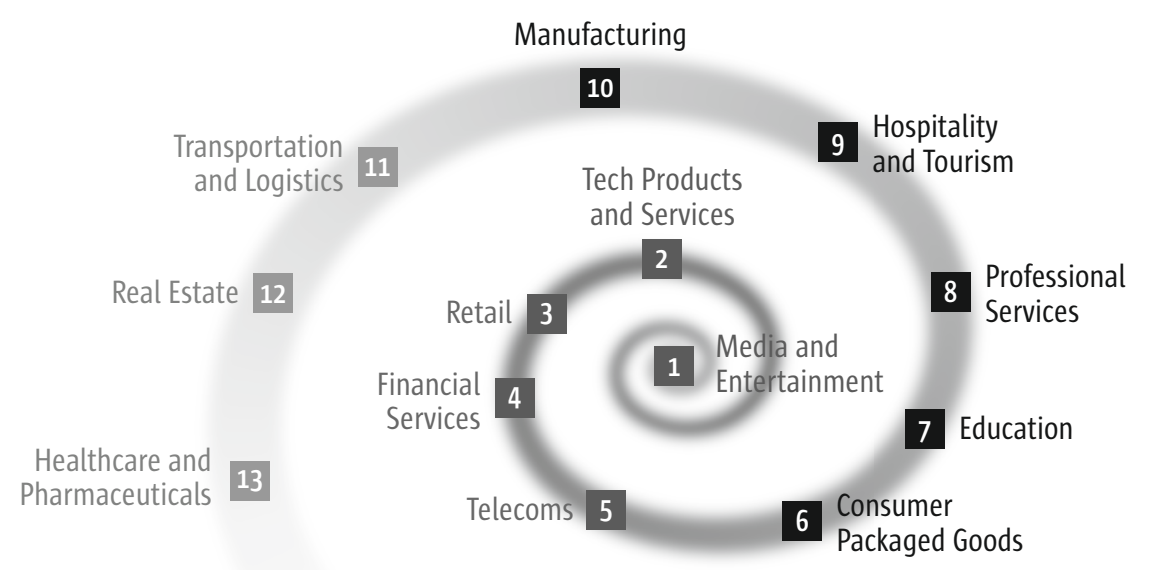

Energy and

Utilities

Abb. 1 Die Grafik zeigt die unterschiedlichen Startzeitpunkte der Branchen im Kontext der Digitalisierung. Sie bedeutet vor allem für die Leistungsträger im Gesundheitswesen einen Zeit- und Wissens-Gap (Life in the Digital Vortex. International Institute for Management Development; Global Center for Digital Business Transformation (c) 2017, www.imd.org).

Dafür sollten bestenfalls alle modernen und möglichen Kommunikationswege rund um die Uhr (24 Std/7 Tage) zur Verfügung stehen. Zusätzlich erwartet der Versicherte von seiner Kasse, ihn unterstützende Gesundheits-Apps, eine (eigentlich selbstverständliche) Vernetzung aller für ihn maßgeblichen Gesundheitsakteure und das alles zu einem möglichst akzeptablen Preis. Ein über dem Durchschnitt liegender (Zusatz-) Beitrag wird nur dann akzeptiert, wenn ein damit verbundener Mehrwert persönlich erkennbar ist. Z.B., die Erwartungshaltung, dass die Krankenkasse die Apps finanzieren, die dazu dienen, dass Krankheiten frühzeitig erkannt, ggf. verhindert oder besser gemanagt werden können.

\section{Transformationsprozess und Handlungsschwerpunkte (Chancen und Risiken)}

Das alles stellt die Krankenkassen vor ungeahnte strategische Herausforderungen und Risiken. Aber sie hält auch ebensolche Chancen bereit, die es mit den damit verbundenen Vorteile für die Kassen, ihre Kunden und Mitarbeitern zu nutzen gilt. Die Krankenkassen müssen deshalb ihre strategischen Positionen systematisch überprüfen und schärfen. Darauf müssen sie ihre Geschäftsmodelle konsequent ausrichten. Damit können beispielsweise Zielgruppen erreicht werden, die bisher nicht erreichbar waren oder es werden digitale Lösungen kostengünstiger möglich. Nur so bleiben sie angesichts des politischen, regulatorischen, demografischen und medizinisch-technologischen Wandels im Gesundheitswesen effizient.

Welche Faktoren dabei eine Rolle spielen, verdeutlichen die folgenden beispielhaft aufgeführten Fragen, die sich die Kassen stellen sollten:

- Was bedeutet die Digitalisierung für unsere Organisation? 
- Wie gestalten wir zukünftig unsere Kommunikation nach Innen und Außen?

- Wie können wir agil auf veränderte Kundenbedürfnisse reagieren?

- Welche Unternehmenswerte müssen unsere Kultur tragen, um zukünftig handlungsfähig zu sein?

- Welche Instrumente der Personalführung benötigen wir, um unsere Führungskräfte und Mitarbeiter als entscheidender Schlüssel für die digitale Transformation zu motivieren?

Ein wichtiger Baustein dazu stellt die Telematik-Infrastruktur dar. Mit der TelematikInfrastruktur, in deren Zentrum die elektronische Gesundheitskarte (eGK) steht, werden 200.00o Ärzte und Zahnärzte, 2.ooo Krankenhäuser, 26.ooo Pflegeeinrichtungen, 20.00o Apotheken, 1.150 Rehabilitationseinrichtungen und 2,3 Millionen Beschäftigte im Gesundheitswesen sowie 70 Millionen gesetzlich Versicherte miteinander vernetzt. Betrachtet man die aktuelle Situation, so sind die verschiedenen Initiativen und Aktivitäten oftmals regionalisiert und entspringen den jeweiligen Versorgungssektoren. Das seit 2005 auf politischer Initiative basierende Vorhaben zur Einführung einer elektronischen Gesundheitskarte ist jedoch ins Stocken geraten. Aufgrund der Schnelligkeit der digitalen Bedarfe und Angebote/Produkte ist ein schnelles Agieren von Seiten der Kassen zwingend. Die Schaffung der Voraussetzungen der Interoperabilitäten der Systeme und Systembeteiligten zur Nutzung derTelematik Struktur und der eCK - auch um ungehemmten Wildwuchs von Systemen zu vermeiden - ist eine für den Gesundheitsmarkt spezifische Notwendigkeit und sollte deshalb politisch gefordert und gefördert werden. Dabei ist auch die zunehmende Nutzung neuer Techniken und mobiler Geräte wie z.B. das Smartphone durch die Kunden sowie deren konkreter Bedarf - z.B. das Erfassen von Life Style-Anwendungen - zu berücksichtigen .

Während sich die Telematik-Infrastruktur im deutschen Gesundheitswesen noch im Aufbau befindet, kommen beinahe täglich neue kommerzielle digitale Gesundheitsanwendungen - überwiegend von großen Smartphone-Anbietern oder globalen Konzernen - auf den Markt. Dabei handelt es sich überwiegend um „Life-Style“-Apps, die ergänzende Informationen und gesund heitsbewusstes Verhalten erzeugen sollen. Hier gilt es für die Kassen, die Spreu vom Weizen zu trennen. Die Anwendungen dürfen nicht in erster Linie der Kommerzialisierung von Daten dienen, sondern sollten

- Schnittstellen überwinden, sektorale Versorgungsstrukturen verbinden und damit die Versorgungslandschaft für die Patienten transparenter gestalten,

- die Zugangsmöglichkeiten zu medizinischer Versorgung und Beratung erleichtern und vereinfachen sowie

- die Erreichbarkeit und den Service von Krankenkasse und Behandlern verbessern.

Gerade bei den mobilen medizinischen Anwendungen/Apps, die durchaus sowohl diagnostisch als auch therapeutisch sinnvoll sein können, stoßen jedoch agile und innovative Verfahren und Anwendungen auf die Anforderungen der „Evidenz“.

Um Innovationen, die diese Kriterien erfüllen, den Zugang in die gesetzliche Krankenversicherung zu erleichtern, müsste allerdings das Regelwerk des Sozialgesetzbuches (SCB) 5. Buch um Erprobungsräume erweitert werden. Auf diesem Wege könn- 
te z.B. das Fernbehandlungsverbot zugunsten telemedizinischer Angebote gelockert oder aufgehoben werden. Gerade angesichts des demografischen Faktors und der damit verbundenen Herausforderungen für eine gute medizinische Versorgung bietet die Telemedizin vor allem in den ländlichen Regionen neue Möglichkeiten, die insbesondere auch älteren und chronisch kranken Menschen zu Gute kommen.

Im Mittelpunkt aller Überlegungen, Aktivitäten und Prozesse muss jedoch der Mensch (Kunde, Versicherte, Patient) stehen. Die Stärkung der Gesundheitskompetenz der Versicherten zählt zu den großen Chancen aber auch Herausforderungen der Digitalisierung. Ein wichtiger Aspekt dabei ist die Vermittlung von seriösem, objektivem und wissenschaftlich gesichertem Wissen. Zahlreiche über das Internet verbreitete Gesundheitsinformationen sind jedoch qualitativ unzureichend bzw. wissenschaftlich nicht gesichert oder ausschließlich von kommerziellen Interessen geleitet. Medizinische Laien können noch weniger als professionelle Nutzer (z.B. Ärzte) die Seriosität der Informationen im Internet interpretieren, geschweige denn prüfen. Umso wichtiger ist es, für höhere Transparenz sowie nutzerorientierte und qualitätsgeprüfte Information zu sorgen. Dazu sollten internetbasierte Gesundheitsportale ausgebaut werden, wie etwa „gesundheitsinformation.de“ des IQWIC (Institut für Qualität und Wirtschaftlichkeit im Gesundheitswesen).

Um Versicherten bei der Nutzung von digitalen Anwendungen (Apps) Sicherheit zu geben, müssen diese als Medizinprodukte zertifiziert werden und die entsprechenden Anforderungen an Qualität und Sicherheit erfüllen.

Im Gleichklang zur Gesundheitskompetenz gilt das informationelle Selbstbestimmungsrecht. Der Versicherte muss „Herr“ seiner Daten bleiben. Das informationelle Selbstbestimmungsrecht hat höchste Priorität und muss für alle Akteure gelten. Der Versicherte wird innerhalb der Digitalisierung zwangsläufig in die Mitte rücken. Denn der Kern einer digitalen Versorgung ist das Vorliegen aller relevanten Daten in plausibler Form. Und der Einzige, der alle Daten zusammenführen darf - und dürfen sollte ist der Versicherte selbst. Nur er entscheidet, wem er welche Daten zur Verfügung stellt. Nicht die Krankenkasse, nicht der Arzt oder das Krankenhaus, nicht der App-Anbieter. Im Idealfall profitieren alle beteiligten Akteure. Der Versicherte durch die Gesundheits-App und verbesserte Prozesse und Versorgung. Der Leistungserbringer durch einfachere und strukturierte Kommunikation. Der Kostenträger durch leitliniengerechte Versorgung seiner Versicherten.

\section{Blick in die Zukunft und Fazit}

Die Leistungsfähigkeit und Effizienz des deutschen Gesundheitswesens, seine Strukturen, Steuerungsinstrumente, seine gesundheitsbezogenen Ergebnisse, aber auch die Fähigkeit sich auf verändernde dynamische Entwicklungen einzustellen, werden aktuell mehr denn je gefordert. Denn mehr und mehr wird Gesundheit vor dem Hintergrund der demografischen Entwicklungen als wichtige Ressource und als ein zentraler Standortfaktor angesehen, der Wachstum, Produktivität und gesellschaftliches Wohlbefinden nachhaltig positiv beeinflusst.

In Zukunft wird das Internet noch stärker als bisher das Leben und Arbeiten der Menschen durch virtuelle Vernetzung sowie flexible Kooperations- und Organisationsformen durchdringen. Reale und virtuelle Welt verschmelzen miteinander, man spricht vom „Internet der Dinge“. 
Im betrieblichen Kontext bestehen neue Möglichkeiten insbesondere durch Optimierung interner und externer (Kommunikation-)Prozesse. Heute schon und in Zukunft noch stärker wird der komplette Geschäftsprozess durch wissensbasierte Systeme unterstützt, mit deren Hilfe Mitarbeiter jederzeit und von jedem Ort aus auf Daten und Information zugreifen können und so auch neues kontextbezogenes Wissen erzeugen. Mit diesen Systemen und einem entsprechenden Wissensmanagement ist es möglich, in Echtzeit Prozesse und ganze Unternehmen zu steuern. Die zunehmende Informationsflut wird damit beherrschbar gemacht, interdisziplinäre Entwicklungen werden vereinfacht. Ebenso ist es möglich, Entscheidungen rechnergestützt zu modellieren um Flexibilität und Beschleunigung zu erreichen. Cloud Computing ermöglicht eine neue Form des Outsourcings und erzielt Kostenvorteile bei gleichzeitiger Steigerung des Leistungsumfangs. Durch die Vernetzung von Datenbanken können alle Arten von Daten ausgetauscht werden (Abele u. Reinhart 2011, BDI u . U_punkt GmbH 2011, Hofmann et al. 2007).

Viel versprechende Entwicklungsmöglichkeiten liegen auch in der Verbindung von Diagnostik und Therapie mit deutlichen Querbezügen zu einer auf die Medizintechnik bezogenen individualisierten Medizin.

Damit die Digitalisierung gerade im Sinne des Versicherten in Form von besserer Versorgung, zielgerichteter Diagnostik, einfacherer Handhabung und bezüglich einer höheren Sicherheit nutzbar gemacht werden kann, sind die Handlungsparameter zu modernisieren.

Dazu bedarf es, die fehlende Interoperabilität der bundesweiten Systeme durch einheitliche Vorgaben zur Entwicklung von weiteren Anwendungen und der Nutzung der telematischen Infrastruktur zu akzelerieren und auch den notwendigen Datenschutz am konkreten Versorgungs- und Kundenbedarf auszurichten.

\section{Die Ergebnisse der Bestandsaufnahme verdeutlichen sowohl die Poten- ziale als auch die Risiken der Digitalisierung im Gesundheitswesen. Mit der Schaffung eines geeinigten Rahmens kann der Gesetzgeber die Entwicklungen zielgerichtet und nutzengesteuert forcieren und maßgeblich den Gesundheitsstandort Deutschland zukunftssicher auf- stellen.}

\section{Literatur}

Abele E, Reinhart G (2011) Zukunft der Produktion. Herausforderungen, Forschungsfelder, Chancen. München 2011

BDI (Bundesverband der Deutschen Industrie e.V.), Z_punkt GmbH (Hrsg.) (2011) Deutschland 2030 - Zukunftsperspektiven der Wertschöpfung. Berlin. URL: http://www.z-punkt.de/studien/studie/deutschland-2030zukunftsperspektiven-der-wertschoepfung (abgerufen am 25.07.2017)

Bundesfachausschuss der CDU (2017) zur E-Health-Strategie Deutschland: 12 Punkte für ein digitalisiertes Deutschland. Berlin. Stand: Jan. 2017. URL: www.cdu.de/system/tdf/media/dokumente/170125_ehealth_ bfa_gesundheit_digital.pdf (abgerufen am 25.07.2017)

Hofmann I, Rollwagen I, Schneider S (2007) Deutschland im Jahr 2020 - Neue Herausforderungen für ein Land auf Expedition. Frankfurt am Main. URL: http://www.dbresearch.de/PROD/DBR_INTERNET_DE-PROD/ PROD0000000000209595.PDF (abgerufen am 25.07.2017) 